\title{
Lectura muda y analgesia del cuerpo olvidado: estudio sobre agresividad y lectura en niños de una Institución Educativa de Medellín*
}

\author{
Silent reading and analgesia of the forgotten body: study on aggression and reading \\ in children of an Educational Institution of Medellín
}

\author{
Eugenio Henao Estrada ${ }^{\text {, Gloria María López Arboleda }}{ }^{b}$ \\ ${ }^{a}$ Rector del Colegio Básico Camino de Paz de la ciudad de Medellín. \\ rectoria@fundacionlasgolondrinas.org \\ ${ }^{b}$ Escuela de Educación y Pedagogía de la Universidad Pontificia Bolivariana, Medellín. \\ glorialopez.ar@gmail.com
}

\section{RESUMEN}

El artículo describe los resultados de una investigación terminada que versó sobre la relación existente entre la adquisición de la lectura y las conductas agresivas en un grupo de niños de tercer grado de una Institución Educativa pública de la ciudad de Medellín. El artículo expone que la lectura no solo ofrece ventajas como es sabido, sino también riesgos que pueden tener consecuencias preocupantes en un mundo que cada vez exige vínculos empáticos y asertivos. La investigación acudió a la complementariedad metodológica; de esta forma, el enfoque cualitativo permitió, a partir del estudio de casos múltiple con diseño inclusivo de cinco subunidades de análisis, apoyarse en la observación no participante y la entrevista semi-estructurada para, aplicando el método de la comparación constante, lograr hallazgos novedosos. Los principales resultados permiten proponer una nueva taxonomía de las emociones, que pone de manifiesto formas sutiles y desapercibidas de agresión en la escuela.

Palabras claves: Emociones, con-tacto, riesgos, lectura.

\begin{abstract}
The article describes the results of a completed research that dealt with the relationship between the acquisition of reading and aggressive behavior in a group of third-grade children from a public educational institution in the city of Medellín. The article states that reading not only offers advantages as is known, but also risks that can have worrisome consequences in a world that increasingly demands empathic and assertive links. The research turned to methodological complementarity; in this way, the qualitative approach allowed, based on the multiple case study with an inclusive design of five subunits of analysis, to rely on non-participant observation and the semi-structured interview to apply the constant comparison method to achieve novel findings. The main results allow proposing a new taxonomy of emotions, which reveals subtle and unnoticed forms of aggression in school.

Key words: Emotions, with-touch, risks, reading.
\end{abstract}

\footnotetext{
* Investigación finalizada en el marco de la Maestría en Educación de la Universidad Pontificia Bolivariana, Medellín. La investigación se titula: Los riesgos de la lectura muda y la analgesia del cuerpo olvidado: investigación a propósito de la relación existente entre la adquisición de la lectura y la manifestación de conductas agresivas en niños de un colegio de la ciudad de Medellín (2018).
} 


\section{INTRODUCCIÓN}

Esta investigación indagó en el vacío que hay entre la relación de la transformación de una habilidad cognitiva, en este caso la lectura, y el desarrollo emocional manifestado en el control de conductas agresivas, partiendo de que el lenguaje es pensamiento y teniendo en la cuenta que la emoción es otra forma de pensamiento y de lenguaje. Lo dicho anteriormente sirve de marco para comprender lo que se propuso la investigación: inferir la relación existente entre la adquisición de la lectura y el control de conductas agresivas en los niños y niñas del grado tercero del Colegio Básico Camino de Paz de la ciudad de Medellín.

El trabajo parte de no asumir la lectura solo como la adquisición de un código escrito sino, además, como la transformación del cerebro y la mente (Dehaene, 2007, citado en Wolf, 2011), es decir, un interés por la forma en que el otro está desarrollando su capacidad cognitiva a través de la lectura y con ello su inteligencia emocional al aceptar que la emoción es otra forma de pensamiento y de lenguaje (Nussbaum, 2008).

Además, el objetivo de la investigación planteó nuevas formas de abordar el aprendizaje desde el sujeto y ofreció una forma diferente de pensar un problema, que tradicionalmente se ha considerado en una sola vía, esto es, la relación entre la emocionalidad como requisito para acceder a la lectura; esta nueva mirada, ofrece la posibilidad de pensarlo como un Moebius, donde hay un continuo entre lectura y emoción en doble vía, el que llevó a pensar que la adquisición de la lectura podía transformar el desarrollo emocional de un sujeto. Lo anterior implica una mirada ética y política a un tema que durante mucho tiempo ha sido objeto de estudio de la educación: la relación entre lectura y convivencia, en la que se reconoce la relevancia de la adquisición de la lectura para el desarrollo del sujeto inmerso en un contexto social y por ende político. Esta investigación propone pensar esta relación desde otra óptica toda vez que entiende que la lectura por sí sola no basta, ya que para lograrlo requiere de la conversación.

Ahora bien, escritura y lectura como un proceso continuo, transforman las estructuras del cerebro y con esto el pensamiento (Dehaene, 2007, citado en Wolf, 2011). Se afirma incluso que solo la mente alfabetizada tiene la capacidad de analizar, clasificar y abstraer mientras que la mente oralizada totaliza; por tanto, investigadoras como Michèle Petit (2008) afirman que,

la escritura no puede limitarse a la transcripción de un código, que cumple una función utilitaria, pues en la medida que transforma la manera de comprender el mundo y de poder proyectar sus ideas al exterior es "un derecho vital como el agua" (p. 1).

Justamente, allí también radican sus riesgos, pues una lectura que resulta tan atractiva y reparadora, puede subsumir al sujeto, atraparlo en un mundo imaginario más atractivo que el real, al punto de llegar incluso a sentir desdén por el plano físico, y por todo lo que tiene que ver con él: el cuerpo (tanto el propio como el del otro), la interacción social y con esto el detrimento de emociones como la compasión que se sustentan en la conversación, en el encuentro.

Por tanto, se planteó en la investigación la cuestión de investigar la emoción para acceder a la adquisición de la lectura en niños y niñas. Aunque, esto cambió, al comprender que:

la lectura traspasa la lengua y tiene implicaciones sociales, culturales y pedagógicas; pues la lectura más que la interpretación del código escrito es una forma de estructurar 
la mente y no se consigue con ninguna otra actividad, ya que la mente oral totaliza y la mente alfabetizada analiza (Marmolejo, 2016).

A partir de ahí, hubo un cambio en la forma de entender la lectura y su incidencia en la transformación de la mente con la pregunta: ¿la adquisición de esta no producirá un efecto similar en la transformación de las emociones, debido al estrecho origen que comparten pensamiento y emoción en todas las áreas del cerebro y el análisis tan profundo que autoras como Martha Nussbaum (2008) han desarrollado al respecto? Luego de esto, inevitablemente la pregunta investigativa se invirtió, de la emoción como condición para acceder a la lectura, a esta como condición para acceder al desarrollo emocional.

Así pues, se inició una indagación informal con siete docentes de Básica Primaria, a las que se les preguntó de manera individual, si con los niños y niñas que no sabían leer -que ellas habían trabajado a lo largo de su experiencia profesional-, consideraban que había algo diferente en sus emociones primarias relativo al control, la comprensión o la manifestación de estas. Todas las docentes manifestaron que sí, y al preguntar en cuál de esas emociones consideraban que ello era más evidente, seis docentes respondieron la ira y el miedo y una respondió la ira y la tristeza; con base en estas respuestas se decidió entonces delimitar el problema a la relación entre la adquisición de la lectura con el control de conductas agresivas.

Lo anterior, llevó al planteamiento de la siguiente pregunta: ¿Podría pensarse entonces, que la no adquisición de la lectura está relacionada con las conductas agresivas en los niños? Esta pregunta ofreció una óptica diferente a la opinión tan generalizada, que afirma que la desmotivación y apatía que genera el no poder comprender los temas académicos, producto del analfabetismo, es la causante de muchas conductas disruptivas que llevan a los niños y niñas a irrumpir, con acciones díscolas o violentas, la armonía necesaria dentro del aula de clase. La pregunta anterior cobra mayor relevancia si tenemos en la cuenta que

la solución de los problemas que tiene el mundo respecto de la corrupción, la delincuencia, la criminalidad y la violencia es la educación. Hay muchas pruebas de que esto es así. Un informe de Unicef de 2005 [...] dice que el 80 por ciento de los presos de todas las cárceles del mundo son analfabetos o semianalfabetos. Del mundo, no de Latinoamérica u Oriente. Yo trabajo en una cárcel en México, donde vivo. El 90 por ciento de los presos que durante su encarcelamiento terminan la preparatoria o empiezan una carrera universitaria, cuando salen no vuelven a delinquir. Un 75 por ciento de los presos que no estudian vuelven a la cárcel (DPA, 2016).

Ahora bien, en la separación tajante que en Occidente se hizo entre cognición y emoción podría radicar la explicación por la que solo hasta ahora la emoción ha comenzado a tener visibilización en el campo de la investigación. Expresado en palabras de Luis Carlos Restrepo (1997) en su libro El derecho a la ternura, “[...] La separación entre razón y emoción es producto de la torpeza y analfabetismo afectivo" (p. 37).

En este orden de ideas, al existir una relación entre la lectura y el desarrollo emocional, no acceder a la alfabetización no solo sería una vulneración del derecho a la educación, sería también una vulneración del derecho a la igualdad, pues con la no accesibilidad a la lectura no solo se podría estar coartando la comprensión de un código, podría significar una afectación de la manera de acceder analíticamente al mundo y de la forma de relacionarnos con los demás. Expresado en palabras de Luis Carlos Restrepo (1997): 
Estamos acostumbrados a reivindicar y opinar sobre los grandes derechos de la vida pública: libertad, trabajo, vivienda, educación, sufragio, sanidad [...] Pero no hablamos casi nunca de los derechos de la vida cotidiana que están confinados a la esfera íntima. Derechos no menos importantes, no menos necesarios. A esta categoría de derechos relegados, casi vergonzantes, pertenece el derecho a la ternura (p. 2).

Finalmente, para concluir esta breve introducción, habría que decir que mucho se ha estudiado el afecto en la adquisición de la lectura, tal vez sea el momento de comenzar a pensar la lectura y sus vínculos con la adquisición del afecto.

\section{MÉTODO}

En términos generales, la investigación acudió a la complementariedad metodológica; de esta forma, el enfoque cualitativo permitió, a partir del estudio de casos múltiples con diseño inclusivo de cinco subunidades de análisis, apoyarse en la observación no participante y la entrevista semi-estructurada para, aplicando el método de la comparación constante, lograr hallazgos novedosos.

Las cinco subunidades de análisis estuvieron conformadas por niños con adquisición de lectura, niños sin adquisición de lectura, padres de niños con adquisición de lectura, padres de niños sin adquisición de lectura y maestros de ambos; la elección de las subunidades se basó en el propósito de inferir más claramente la relación de las categorías al utilizar de manera complementaria, el método de comparación constante de la teoría fundamentada (Hernández et al., 2006), lo que permitió a su vez, comparar datos con datos, categorías con categorías, datos con teoría y categorías con teoría.

Ahora bien, el diseño específico posibilitó estudiar varios casos únicos (Hernández et al., 2006) que permitieron a su vez inferir la relación entre adquisición de la lectura y manifestación de conductas agresivas, teniendo en cuenta que cada caso pudo aportar a la comprensión global del problema, y con esto favorecer la capacidad de contrastar las respuestas de cada subunidad de análisis. Se utilizó la observación no participante en el campo con el fin de contrastar lo observado por los investigadores con las entrevistas individuales semi-estructuradas (Hernández et al., 2006) realizadas a padres y maestros. Diversos documentos institucionales también sirvieron de apoyo para la obtención de información. Para la realización de las entrevistas semi-estructuradas, se construyeron dos tipos de instrumentos (guías de entrevista), uno para padres y otro para maestros. Por su parte, los formatos de consentimiento informado sirvieron como documentos que presentaron las condiciones éticas de la investigación, que incluyeron el anonimato y la libre participación de los sujetos investigados (Hernández et al., 2006).

La muestra fue heterogénea, de participantes voluntarios y por conveniencia. Se acudió además a la muestra de caso tipo (Hernández et al., 2006) donde se profundizó en la información de un segmento de niños de un colegio de la comuna ocho de Medellín con y sin adquisición de la lectura, sus padres y maestros, como se dijo anteriormente; se usaron criterios de inclusión como pertenecer al mismo grupo escolar, contar con los mismos maestros, las condiciones familiares, socioeconómicas y culturales similares, pero con niveles de adquisición de la lectura diferentes, como ya se ha señalado antes. 
En cuanto a la muestra de participantes voluntarios (Hernández et al., 2006) se contó con un consentimiento informado de los padres de familia para la intervención con los niños, así como la participación voluntaria de los maestros y padres; la muestra por conveniencia (Hernández et al., 2006) incluyó el hecho de que la investigación se desarrolló en el lugar de trabajo de uno de los investigadores, lo que viabilizó el asunto porque hubo un acercamiento previo y empático con los participantes de la investigación. En total, se trabajó con una muestra compuesta por tres estudiantes, tres madres y dos maestras.

El proyecto investigativo se acogió además, a la resolución 8430 de 1993 del Ministerio de Salud, donde se postulan las normas para la investigación científica, la que, en consonancia con la declaración universal sobre bioética y derechos humanos de la Unesco (2005), se respetaron los principios fundamentales como la salud, la integridad, la vida, la dignidad, intimidad, autodeterminación y confidencialidad de la información de cada uno de los participantes de la investigación, a quienes se les explicó de forma escrita y verbal de qué se trataba su participación en esta.

\section{RESULTADOS}

Esta investigación, en concordancia con lo planteado por los diferentes teóricos hasta aquí nombrados, considera que las emociones parten de la mente y pasan por el cuerpo de manera inconsútil. En los casos en que no hay adquisición de la lectura, las emociones se manifiestan a través del cuerpo, de manera considerada políticamente inadecuada, grotesca, vulgar, pues el cuerpo está asociado a emociones de asco, vergüenza e impudor. Además, el cuerpo históricamente ha sido considerado con un estatus inferior a la mente, por tanto, muchas de sus manifestaciones se consideran primarias y socialmente inapropiadas; es el caso de los estudiantes que agreden físicamente, gritan y deben estar en constante movimiento, por esto, en esta investigación se ha denominado a este tipo de emociones: "actuantes". A este tipo de estudiantes, según manifiestan algunas madres, les gusta hacer llorar al otro. Se entiende el llanto como signo leíble en el cuerpo del otro; la expresión y la comunicación a través del cuerpo.

De otro lado, en el caso de adquisición de la lectura donde el pensamiento alcanza niveles de análisis, clasificación y abstracción, hay un desarrollo de la inteligencia emocional, lo que denota manifestación y control sobre ellas, por tanto, en esta investigación se han nombrado como: "emociones políticamente adecuadas", pues nacen en la mente y el sujeto reconoce las maneras social y culturalmente adecuadas de expresarlas. Sin embargo, son unas emociones inmaduras, incompletas en el reconocimiento del otro como beneficiario de mis cuidados: "la ética se da en el encuentro con el otro" (...). El otro es próximo a mí en tanto yo soy responsable de él" (Levinas, 2000, citado en Magendzo, 2006, p. 7). Es decir, un sujeto con capacidades cognitivas y culturales socialmente apropiadas, pero deshumanizado, indolente del sufrimiento del otro. Dentro de esta clasificación se encuentran los estudiantes que no agreden físicamente, pero tampoco juegan y desde su inactividad ignoran y excluyen al otro, se aíslan y aíslan, siendo esta otra forma de agresión. Es decir, la lectura comprendida desde el utilitarismo y como vehículo de interacción social, aunque no necesariamente como experiencia que toca.

Por último, están las emociones que esta investigación ha llamado "con-tacto"; son las que, gracias a la adquisición de tecnologías como el arte, desarrollan la imaginación para 
alcanzar lo que no se alcanza de manera física directa (Nussbaum, 2008), transforman el pensamiento y la inteligencia emocional y propician la intimidad como espacio para la reflexión sin escindirse del cuerpo, sin menospreciarlo, aislarse, omitirlo, inmovilizarlo ni degradarlo. Es decir, un cuerpo que corre al encuentro con el otro, lo escucha y conversa con él. Por tanto, reconoce al otro como él mismo; se ve en su sufrimiento y se reconoce en su dolor. Un sujeto ético que es capaz de utilizar su cuerpo para ponerse de pie, y gritar ante la injusticia que sufren los otros, pues es consciente de que ellos son él mismo.

Por tanto, las emociones con-tacto requieren no objetivar al otro, sino ser-con-él, poder reconocerse en el otro, ver las emociones propias en el otro de manera especular, en palabras más concretas, comprender que la emoción que el otro genera en mí, se produce, porque él también la está sintiendo, allí radica la empatía, la compasión, la comprensión, la experiencia, en síntesis, la humanización, y para lograrlo es fundamental la lectura, pero ésta a su vez limita al sujeto, cuando no es atravesada por la oralidad.

Finalmente, la oralidad permite tramitar las emociones a través de la palabra, sin necesidad de que se encarnen de manera desbordada en el cuerpo, como emociones actuantes, y en interacción con otro, distinto a la palabra escrita que aísla y en ocasiones deshumaniza manifestándose como emociones políticamente correctas.

\section{DISCUSIÓN}

El sujeto tiene escindida la mente y el cuerpo, por ello quienes leen simbolizan y a través de la simbolización canalizan sus emociones y pueden llegar a expresarlas de un modo socialmente más aceptado, a diferencia de quienes no leen y ante esa ausencia pasan al acto y expresan por el cuerpo: "Ciertamente hemos de conceder que toda experiencia humana se encarna y, por tanto, se realiza en algún tipo de proceso material. De esta manera, todas las emociones humanas son procesos corporales" (Nussbaum, 2008, p. 81). Podría pensarse en unos procesos mentales encarnados en el cuerpo.

Por lo tanto, la lectura también ofrece ciertos riesgos, como pudo evidenciarse en los testimonios de esta investigación, a su vez que el texto puede convertirse en una prótesis del cuerpo, una prótesis que potencia la imaginación y lo lleva a desplazarse a otros mundos, espacios o lugares desde la quietud de un sofá, sin necesidad de interactuar con otro, es decir cuando la lectura no es una experiencia mediada por el encuentro con el otro lleva al aislamiento como sucedía con los eremitas, o en algunas ocasiones la lectura ha ofrecido un poder, un estatus que lleva a aislarse y a excluir al otro pues se considera que no está a la altura del interlocutor.

De este modo, el cuerpo y su inteligencia propia quedan relegados y aparece la parálisis del cuerpo, una quietud que solo deja espacio para leer y no cultivar el principio griego de mente sana en cuerpo sano, al entender el cuerpo como un objeto frívolo y banal que incluso se descuida como una simple extensión de la, en ocasiones sobrevalorada, mente, como se ha creído en Occidente a partir de los planteamientos de Descartes. Es por esto que puede decirse que,

Los especialistas tienen joroba, porque viven en espacios angostos, de techos bajos y frecuentemente mal ventilados, que tienen el culo gordo debido a su vida sedentaria [...] un cuerpo torpe y que no se expone, de un cuerpo que solo sabe cumplir metódica, 
segura y regularmente con sus deberes de asignatura, con sus tareas asignadas (Nietzsche, 1863, citado en Larrosa, 1999, p. 20).

Sin embargo, los primeros aprendizajes se obtienen a través del cuerpo; los antepasados, por ejemplo, aprendían haciendo, razón por la que el cerebro presenta mayor activación y disposición al aprendizaje cuando inicia el movimiento, según trabajos recientes en neurociencias. Sin embargo, la educación actual pretende la enseñanza desde la inmovilidad. Tanto así, que, en las entrevistas realizadas a maestros y padres en el transcurso de esta investigación, surgió que a los niños que leen deben invitarlos a jugar, porque el estudio ha suplido la función del juego.

Estudiar entonces es inmovilizarse; el cuerpo se relega, con esto se olvida conversar con el otro y sin conversación no se construye empatía por más conocimientos que se tengan. Larrosa hace alusión a la importancia del cuerpo en la comprensión del lenguaje, y para esto cita a Nietzsche, quien aseguraba que para comprender el cuerpo de las palabras se necesitaba de otro cuerpo, es decir el lenguaje; el pensamiento y por tanto las emociones parten de la mente, pero pasan por el cuerpo: "En rigor solo se puede amar un cuerpo, siendo un cuerpo, a través de un cuerpo, haciendo cuerpo con un cuerpo, cuerpo a cuerpo, entre los cuerpos" (Nietzsche, 1863, citado en Larrosa, 1999, p. 21).

Es por eso, que leer marca una diferencia significativa en el relacionamiento con el otro cuando es una experiencia, según Larrosa, de lo contrario, leer al igual que no hacerlo no solo no desarrolla empatía, también hay manifestaciones de conductas agresivas; se explica así, lo que se ha denominado en esta investigación los riesgos de leer.

Por otro lado, están quienes no han accedido a la lectura y expresan a través del cuerpo, bien sea mediante la agresión física hacia al otro, a través de la somatización, del grito (como manifestación que escapa al lenguaje verbal y es un punto intermedio entre la oralidad y la expresión del cuerpo).

Es decir, quienes leen simbolizan, tramitan su manifestación de conductas agresivas a través de su mente pues el libro es una prótesis de su cuerpo, mientras que quienes no leen no tienen esta prótesis, el cuerpo es su instrumento y manifiestan su agresividad a través de él, lo cual en una sociedad que sobrevalora la razón y se avergüenza de su cuerpo, es mirado con desdén y prejuicio (cualquier manifestación corporal debe ser tratada en privado); por tanto, las manifestaciones de conductas agresivas desde la simbolización son socialmente mejor aceptadas, más sutiles; así pues, se puede excluir y agredir al otro siempre y cuando no sea a través del cuerpo, de manera física, sino simbólicamente; por tanto, quien ignora al otro, no lo siente a su mismo nivel de comprensión, no conversa con él, le rehúye y finalmente lo aísla, es socialmente más aceptado que quien con su cuerpo lo estruja para alejarlo. Igual que aquel que no se desplaza a conocer a Venecia, pero a través del cuerpo-libro la recorre, la imagina, escapa a su realidad y viaja a ella en ese cuerpo prestado, a diferencia de quien la camina con sus pies y la toca con sus manos.

Es necesario pues que la lectura no sea una actividad, sino una experiencia que toque al sujeto y le permita ser tocado a través del encuentro con el otro; que al leer se lea, se comprenda para que pueda leer y comprender al otro. Muchos leen, pero pocos tienen comprensión entre líneas de lo que leen. Cuando la lectura posibilita la conversación (Larrosa, 2006), trasciende lo utilitario del código, va más allá del mero desarrollo del pensamiento, y entonces, la lectura, deja de ser una herramienta de poder y se convierte 
en un instrumento para desarrollar la compasión. Si la lectura transforma la mente, como lo aseguran autores como Stanilas Dehane o Walter Ong, y la emoción es pensamiento, entonces la lectura debe transformar el desarrollo emocional. Pero no basta con leer para una manifestación de emociones adecuada, es necesario que la lectura sea un encuentro con el otro que, finalmente, es él mismo (Carmona, 2015); porque para poder trascender de la lectura racional a la empatía, es necesario ser consciente de que el otro es una continuidad del sujeto, de que lo otro es lo mismo.

En este orden de ideas, y de acuerdo con la figura de Moebius, la representación lingüística de ser tocado por la experiencia de leer permite que el libro no sustituya al cuerpo, no aislarse, y correr al encuentro del otro para conversar, para reconocerlo y comprenderse en él; pues sólo ahí habrá un desarrollo de las emociones a través de la lectura, una construcción del sujeto y una organización del caos interior que no se encuentra en el conocimiento de la información que contiene el libro, por ejemplo, las experiencias fundacionales de la vida, las preguntas verdaderamente significativas:

Me refiero obviamente a las situaciones que hacen referencia a la contingencia, es decir, a aquellas preguntas que implican la vida humana en su totalidad o, mejor todavía, el sentido de la vida: el nacimiento, el amor, el mal, el sufrimiento, la muerte [...] situaciones que no pueden resolverse acudiendo a la figura del experto. (Bárcena, Larrosa y Mélich, 2006, p. 254).

Como se dijo, estas, no están en el contenido de los libros, pero sí están en la lectura porque ésta es el lugar de encuentro entre dos sujetos; si la lectura se enmarca desde una posición, de poder desde el saber, se obtendrán niños que quizá no griten, no corran, no interrumpan, no agredan físicamente, y sin embargo, aunque está manifestando sus emociones de manera políticamente correcta, no quiere decir esto, que internamente haya armonía (Petit, 1999), pues en la medida que la experiencia de leer no es con relación al otro, sino con metas individuales, esta no aporta a la comprensión del otro para ser empático, para no excluirle, para no dar más valor a las competencias que a las capacidades (Nussbaum, 2010), para no formar individuos que privilegien el saber sobre el ser; tal vez, el prejuicio de que todo lector es una persona sensible y culta, es el que ha sostenido la idea de que la razón está por encima de la emoción.

En conclusión, leer, más que una tecnología es una experiencia, y es fundamental en el desarrollo mental y emocional del sujeto, pero a diferencia del pensamiento, donde con solo adquirir el código transforma la capacidad de analizar, clasificar y abstraer, en lo emocional no basta con leer para desarrollar empatía, compasión o ética, pues es necesario, en todo caso, que la lectura sea una conversación con el otro, no un evento que se restringe al aislamiento y a la inmovilidad del cuerpo, sino un encuentro, con el cual puedo conversar sin interesar si es un analfabeto o un alfabetizado:

[...] "sin embargo el que la lectura y la escritura no se problematicen de manera explícita, el hecho de que, al menos en apariencia, ni la lectura ni la escritura sean un problema, no significa que no sean el lugar de potentísimos mecanismos de control. Si no fuera por esos mecanismos sería imposible la imposición generalizada y la posterior naturalización de esa lengua de nadie" (Larrosa, 2006, p. 33). 
Pocas veces se habla sobre los riesgos de la lectura y la escritura, pero, como lo expresa Larrosa, pueden ser un mecanismo de control, que ahoga nuestra propia lengua, nuestro propio criterio, y al guiar la forma de pensar del otro, no se puede concebir como una intelectualidad al servicio de la ética, ni de la empatía.

Es decir, no basta con leer la lengua de todos, pues si no se construye una lengua propia, en conversación con el otro, a quien se escucha y a quien se comprende, difícilmente emociones como empatía, compasión o amor pueden desarrollarse; si la lectura no es una experiencia de conversar con otro, o sea, si la lectura no pasa por el cuerpo, no como grito sino como conversación, difícilmente se tendrá comprensión lectora de la vida, del otro y del propio yo, como muy apropiadamente lo dice Larrosa: "no queremos que se nos comprenda, sino que se nos escuche, y somos capaces de ofrecer, a cambio, nuestra capacidad para escuchar lo que quizá no comprendemos" (Larrosa, 2006, p. 37).

Por consiguiente, Larrosa afirma que la experiencia no es acción sino pasión, es la reflexión de cada uno sobre sí mismo, si no leo para elaborar con otros, y eso que leo no me toca ni me permite tocar a otros, entonces estoy leyendo sólo para mí, una lectura utilitaria, y desde allí no se construye un desarrollo emocional a través de la lectura, por más que se tenga un dominio sobre el cuerpo que ya no grita, no corre y no agrede, pero tampoco conversa y se aísla: "Necesitamos un lenguaje para la experiencia, para poder elaborar con otros" (Bárcena et al., 2006, p. 255). La experiencia es la reflexión a través de la pasión y no de la acción, pero la aplicación de esa experiencia es poder tocar y ser tocado por la lengua del otro, y para ello es necesaria la acción, el cuerpo:

Cuando se trata de considerar la lengua desde su relación con el cuerpo y con la subjetividad, frecuentemente se apela a nociones que tienen que ver con la oralidad, con la boca y con la lengua, con el oído y con la oreja, con la voz. Y ahí no se trata de la diferencia entre habla y escritura, sino la diferencia entre distintas experiencias de la lengua incluyendo el leer y el escribir [...]. La voz es la marca de la subjetividad en la experiencia del lenguaje, también en la experiencia de la lectura y de la escritura. En la voz lo que está en juego es el sujeto que habla y que escucha, que lee y que escribe. A partir de aquí se podría establecer un contraste entre una lengua con voz, con tono, con ritmo, con cuerpo, con subjetividad, una lengua para la conversación... y una lengua sin voz, afónica, átona o monótona, arrítmica una lengua de los que no tienen lengua (Larrosa, 2006, p. 41).

En otras palabras, para poder hablar una lengua propia, una que permita construir valores y emociones es necesario un cuerpo con boca, oídos, lengua, un cuerpo adaptado para la conversación y, como aclara Larrosa, ello no se limita a la oralidad, también a la lectura y la escritura. Leer, usando el libro como una prótesis del cuerpo, quedándose en el plano imaginario y sin concretarse en la acción, no posibilita convivir empáticamente con otros.

El desarrollo emocional es distinto, necesita del otro, y tal vez es más complejo que el cognitivo. Tal vez es más complejo, porque la emoción es pensamiento en acción, pensamiento que pasa por el cuerpo de manera inconsútil con la mente, distinto al análisis y la cognición que procura habitar una mente fragmentada del cuerpo; por tanto, sin lectura, las manifestaciones emocionales se desbordan en el cuerpo, y cuando hay lectura y simbolización se regulan, pero las emociones no tocan, no hacen con-tacto, porque la 
parálisis del cuerpo y el aislamiento no favorecen el encuentro con el otro, y su ulterior elaboración ética. Es decir, sin lectura se afecta la manifestación política de las emociones, y con lectura la manifestación humana de éstas. Sólo a través de la lectura como experiencia se construye inteligencia emocional y ética.

La diferencia está en que la adquisición de la lectura, como toda tecnología, requiere ser enseñada, requiere de otro de quien aprender, pero una vez se aprende, se puede aislar de esos otros y acarrear los problemas morales que plantea Steiner; en cambio, una vez se adquiere la lectura debe volverse conversación a través del cuerpo: de la lengua, de la boca, del oído, de las cuerdas vocales, de los pulmones, es decir, del encuentro permanente con el otro, y solo así se reconfigura el sujeto del que habla Petit, cuando menciona que alcanza armonía interior para poder ser melodioso con el mundo (Petit, 1999), o desarrollar compasión que hace parte de las capacidades que plantea Nussbaum (2010), recordando además, que el fin utilitario de la lectura se restringe a las competencias.

\section{CONCLUSIONES}

Cuando se dio inicio a esta investigación se consideró la relevancia de la adquisición de la lectura en el desarrollo emocional, pero en la medida que se avanzó, se encontró que, aunque la adquisición de la lectura es muy significativa, si esta no es atravesada por la conversación, acarrea ciertos riesgos como el aislamiento, la subutilización del cuerpo, la pretensión de inmortalidad y una afectación del principio de realidad, en síntesis, la deshumanización.

Así pues, la presente investigación posibilitó la construcción de una clasificación de las emociones en tres tipos: A) emociones actuantes, B) emociones políticamente adecuadas y C), emociones con-tacto (todas ellas con una característica común, ser emociones ubicuas); es decir, los sujetos que no acceden a la lectura, ante su incapacidad de simbolizar expresan a través del cuerpo, bien sea agrediendo a personas o a cosas, auto agrediéndose, somatizando, o a través del grito que es una manera intermedia entre la oralidad, la corporalización y la agresión (denominadas emociones actuantes).

Por otro lado, en las denominadas emociones políticamente adecuadas, las personas que han accedido a la lectura sin mediación con la conversación, por el reconocimiento del otro, pueden ver afectado su principio de realidad, pueden terminar aislándose del contacto con los demás, y hay un fortalecimiento de la intelectualidad en detrimento de su cuerpo. Estas personas pueden agredir desde la exclusión del otro, y su total indiferencia con ellos y con su entorno, siendo incapaces así de desarrollar empatía o compasión por el sufrimiento ajeno.

Finalmente, están las emociones con-tacto, que dan cuenta de la lectura como una experiencia que permite tocar y ser tocado; se han denominado con-tacto por la doble acepción que conlleva la palabra: por un lado hay un contacto entre dos sujetos y por otro, apoyado en la diferencia que se establece entre la pedagogía del tacto y la táctica de la pedagogía; en este caso hay una emoción del tacto, un tacto que permite tocar y ser tocado, ya que, como lo indica la palabra es un toque con un tacto tal que transforma al otro y en ese orden de ideas, solo yo me transformo, nadie me transforma, aunque necesito de otro para transformarme, de lo contrario es imposible.

Por último, las conclusiones pueden contrastarse con tres asuntos fundamentales del planteamiento del problema de esta investigación: el primero, es que la relación entre 
lectura y desarrollo emocional es en doble vía; por tanto no acceder a la alfabetización es una vulneración no solamente del derecho a la educación, también del derecho a la igualdad, pues con la no accesibilidad a la lectura se están viendo afectadas, la manera de acceder analíticamente al mundo, la capacidad de trascender al cuerpo para expresar por medio de la simbolización (Petit, 1999) y la oportunidad de conversar sobre lo leído para alcanzar a desarrollar emociones con-tacto, es decir, la forma de relacionarse con los demás.

Por otra parte, la Banda de Moebius ayudó a comprender que todo sujeto es a su vez objeto (Carmona, 2015), y en esta relación bidireccional este estudio pudo argumentar la relación en doble vía entre lectura y emociones, pues en los antecedentes teóricos e investigativos se encontró abundante información sobre el papel del afecto en la adquisición de la lectura, pero no así, sobre el fenómeno de manera inversa.

Por último, la presente investigación se interesó por estudiar el vacío teórico existente en la relación entre la transformación de las emociones y la manifestación de conductas agresivas mediante la lectura. Sin embargo, la investigación fue más allá en la comprensión del fenómeno, al proponer que la lectura por sí sola no alcanza a desarrollar emociones como la empatía o la compasión, necesarias en el control de conductas agresivas sutiles, como el rechazo, la exclusión o la indiferencia, y que, por el contrario, es un factor de riesgo que las puede propiciar (Steiner, 2013) en la escuela. Ello condujo a los investigadores a la conclusión de que la lectura ofrece ciertos riesgos deshumanizantes (Steiner, 2013), y para evitarlos, debe transitar por la oralidad a modo de conversación (Larrosa, 2006), en aras de alcanzar a desarrollar lo que esta investigación denominó emociones con-tacto.

En síntesis, esta investigación reconoce en la lectura una tecnología que brinda capacidad de análisis, clasificación y abstracción, es decir, modifica el pensamiento (Ong, 2006), o como lo expresa Logan (1987), citado en Marmolejo (2016), solo la lectura amuebla la casa; además, gracias a ella puede encontrarse un pensamiento pausado y reflexivo como afirma Roland Barthes (2009); también es cierto, que la lectura permite expresar emociones de manera simbólica, lo cual evita que, necesariamente, se manifieste desde el pasaje al acto (Petit, 1999); además, invita al recogimiento, favorece habilidades empáticas y mediante la simbolización ayuda a organizar el caos interno del sujeto (Petit, 1999), en tanto eso sí, según el presente trabajo, la lectura posibilite el encuentro con el otro. Sin embargo, contrario a lo que los investigadores suponían al inicio de esta investigación, puede concluirse, además, que la adquisición de la lectura puede traer consigo, no solo las ganancias antes descritas, sino la emergencia de conductas agresivas, las cuales pueden estar encubiertas tras un desarrollo emocional políticamente correcto, asunto que se advierte en esta disertación, no como juicio de valor, sino como potencialidad de transformación, en la escuela en particular, y en la vida en general.

\section{REFERENCIAS BIBLIOGRÁFICAS}

Bárcena, F., Larrosa, J. y Mèlich, J. (2006). Pensar la educación desde la experiencia. Revista portuguesa de pedagogía, 40(1), 233-259. Doi: https://doi.org/10.14195/1647-8614_40-1_11

Barthes, R. (2009). El susurro del lenguaje, Más allá de la palabra y de la escritura. Barcelona: Paidós.

Carmona, J. 2015. El sujeto en la banda de Moebius. Recuperado de https:/es.scribd.com/ document/364760166/1-El-Sujeto-en-La-Banda-de-Moebius-Jaime-Carmona (30-08-2018). 
DPA. (2016). Jorge Bucay: la solución que tiene el mundo a los problemas es la educación. Diario la mañana. Recuperado de http://xn--lamaanaonline-lkb.com.ar/noticia/32455-jorge-bucay-lasolucion-a-los-problemas-que-tiene-el-mundo-es-la-educacion (17-09-2018).

Hernández, R. Fernández, C. y Baptista, P. (2006). Metodología de la investigación. México DF: Mc Graw Hill.

Larrosa, J. (1999). Erótica y hermenéutica, o el arte de amar el cuerpo de las palabras. Educación y pedagogía, XI, 17-27.

. (2006). Una lengua para la conversación. Revista Educación y Pedagogía, 18, 29-42. Recuperado de http://aprendeenlinea.udea.edu.co/revistas/index.php/revistaeyp/article/view/19062/16285

Magendzo, A. (2006). El Ser del Otro: un sustento ético-político para la educación. Polis, Revista Latinoamericana. Recuperado de https://polis.revues.org/4897

Marmolejo, A. (2016). Conversatorio sobre educación. Medellín. Fundación las Golondrinas. 05 de agosto de 2016.

Nussbaum, M. (2008). Paisajes del pensamiento, la inteligencia de las emociones. Barcelona: Paidós.

Nussbaum, M. (2010). Sin fines de lucro, por qué la democracia necesita de las humanidades. Buenos Aires: Katz.

Ong, W. (2006). Oralidad y escritura. Tecnologías de la palabra. Buenos Aires: Fondo de Cultura Económica.

Petit, M. (1999). Nuevos acercamientos a los jóvenes y la lectura. México DF: Fondo de Cultura Económica.

Petit, M. (2008). El arte de la lectura en tiempos de crisis. Barcelona: Océano Travesía.

Restrepo, L. (1997). El derecho a la ternura. Editorial Península.

Steiner, G. 2013. En el castillo de Barba Azul. Barcelona: Gedisa.

Wolf, Ch. (2011). El beneficio encubierto de leer. Mente y cerebro, 47, 36-41. 